\title{
LOBIN: E-Textile and Wireless-Sensor-Network- Based Platform for Healthcare Monitoring in Future Hospital Environments
}

\author{
Gregorio López, Víctor Custodio, and José Ignacio Moreno, Member, IEEE
}

\begin{abstract}
This paper describes a novel healthcare IT platform developed under the LOBIN project, which allows monitoring several physiological parameters, such as ECG, heart rate, body temperature, etc., and tracking the location of a group of patients within hospital environments. The combination of e-textile and wireless sensor networks provides an efficient way to support noninvasive and pervasive services demanded by future healthcare environments. This paper presents the architecture, system deployment as well as validation results from both laboratory tests and a pilot scheme developed with real users in collaboration with the Cardiology Unit at La Paz Hospital, Madrid, Spain.
\end{abstract}

Index Terms-E-textile, healthcare, indoor location, wireless sensor networks (WSNs).

\section{NOMENCLATURE}

\section{AGPS}

BP

DAPB

DP

DSR

ECG

E-OTD

ETB

GSM

GUI

ISM

IT

LBS

LQI

MAC

MTU

Nchannels

Pbeacons

PDA

PCB
Assisted-global position system.

Beacon point.

Data acquisition and processing board.

Distribution point.

Dynamic source routing.

Electrocardiogram.

Enhanced observed time difference.

Ethernet transmission board.

Global system for mobile communication.

Graphical-user interface.

Industrial scientific and medical.

Information technology.

Location-based services.

Link quality indicator.

Media access control.

Maximum transfer unit.

Number of channels available for sending location beacons.

Period BPs send beacons with.

Personal digital assistant.

Printed-circuit board.
Manuscript received February 17, 2010; revised June 5, 2010; accepted July 2, 2010. Date of publication July 19, 2010; date of current version November 5 , 2010. This work was supported in part by the Spanish Ministry of Industry, Tourism, and Trade under the LOBIN project (TSI-020302-2008-57).

The authors are with the Telematic Engineering Department, Carlos III University, Madrid 28911, Spain (e-mail: gregorio.lopez@uc3m.es; victor. custodio@uc3m.es; joseignacio.moreno@ieee.org).

Color versions of one or more of the figures in this paper are available online at http://ieeexplore.ieee.org.

\author{
Ptx_loc \\ RFID \\ RSS \\ SMS \\ TDoA \\ Tlisten \\ ToA \\ UWB \\ WDAD \\ WLAN \\ WSN \\ WTB \\ Period of time WTBs send location information \\ with. \\ Radio frequency identification. \\ Received signal strength. \\ Short message service. \\ Time difference of arrival. \\ Time WTBs spend listening to each channel \\ available for location. \\ Time of arrival. \\ Ultra wideband. \\ Wearable data-acquisition device. \\ Wireless local area network. \\ Wireless sensor network. \\ Wireless transmission board.
}

\section{INTRODUCTION}

INCE its beginnings, science has, in some ways, been devoted to human healthcare, e.g., trying to improve life expectancy or to improve quality of life. Currently, much research is still being carried out in many different fields, such as digital signal processing, electromagnetic engineering, electronics or computer science, to further develop smarter equipment, devices, and systems related to this topic. Furthermore, some consulting studies have recently pointed out healthcare IT as a key investment area to overcome the current difficult economic situation [1].

Most traditional medical solutions, despite being very sophisticated and accurate, do not meet some key requirements for today's as well as future applications within this field. Nowadays, the demand for noninvasive and pervasive healthcare services that impact a patient's normal life as little as possible is increasing dramatically [2].

Advances in nanotechnology and smart materials have lead to electronic textiles (e-textiles) that are able to measure biometric parameters in a noninvasive manner. Using e-textiles, wearable healthcare-monitoring systems can be developed avoiding the use of cables wired around the patient as in current practice.

Communications advances in wireless sensor networks (WSNs) provide a cost-effective solution to support wide coverage and mobility (i.e., pervasiveness) allowing natural movement of patients and potential development of added-value services by developing remote patient control and automatic alert detection in real time based on healthcare measurements. By using this technology, a location service could be deployed, supporting the location of patients as well as staff inside the hospital. 
TABLE I

RELATED WORKS

\begin{tabular}{|c|c|c|c|c|c|c|c|c|}
\hline & $\begin{array}{c}\text { Target } \\
\text { Application }\end{array}$ & $\begin{array}{l}\text { Wireless } \\
\text { Technology }\end{array}$ & $\begin{array}{l}\text { Uealth Status } \\
\text { Monitoring }\end{array}$ & $\begin{array}{l}\text { e-Textile } \\
\text { based }\end{array}$ & $\begin{array}{c}\text { Data } \\
\text { Acquisition } \\
\text { Modes }\end{array}$ & Location & $\begin{array}{c}\text { System } \\
\text { Capacity } \\
\text { Analysis }\end{array}$ & $\begin{array}{l}\text { Validation in } \\
\text { Real } \\
\text { Fnvironments }\end{array}$ \\
\hline AMON [4] & Telemedicine & GSM & Yes & No & Periodically & No & & Yes \\
\hline Life-Guard [5] & Adventurers & Bluetooth & Yes & No & $\begin{array}{l}\text { Locally recorded } \\
\text { Continuously }\end{array}$ & No & & Yes \\
\hline MagIC [6] & $\begin{array}{l}\text { Telemedicine } \\
\text { Clinical } \\
\text { Environments }\end{array}$ & & Yes & Yes & Continuously & No & No & Yes \\
\hline $\begin{array}{c}\text { WEALTHY } \\
{[7]}\end{array}$ & Telemedicine & GPRS & Yes & Yes & Quasi-real-time & No & & Yes \\
\hline CodcBlue [8] & $\begin{array}{c}\text { Hospital } \\
\text { environments }\end{array}$ & WSN (802.15.4) & Ycs & No & Continuously & $\begin{array}{c}\text { Ycs } \\
\text { lndoor } \\
\text { location }\end{array}$ & No & No \\
\hline Smart Vest [9] & High-risk workers & $\begin{array}{c}\text { Proprietary from } \\
\text { Xtream in the } \\
\text { ISM band ( } 2.4 \\
\mathrm{GHz})\end{array}$ & Yes & Yes & Continuously & $\begin{array}{c}\text { Yes } \\
\text { Gco- } \\
\text { location } \\
\text { (GPS) }\end{array}$ & & Yes \\
\hline Lee et al $[10]$ & Sport & WSN $(802.15 .4)$ & Yes & Yes & Continuously & No & No & Yes \\
\hline LOBIN & $\begin{array}{c}\text { I Iospital } \\
\text { environments }\end{array}$ & WSN (802.15.4) & Yes & Yes & $\begin{array}{c}\text { Continuously } \\
\text { On-demand } \\
\text { After any alarm }\end{array}$ & $\begin{array}{c}\text { Yes } \\
\text { Indoor } \\
\text { location }\end{array}$ & Yes & Yes \\
\hline
\end{tabular}

The objective of this paper is to present the results of the LOBIN platform, which provides remote location and healthcare-monitoring support for hospital environments based on the combination of e-textile and WSN technologies.

During the past few years, much research has been carried out in this area and a number of wearable physiological monitoring systems to monitor the health status of the individual wearer have been developed [3]-[10]. Table I summarizes some of the most relevant works compared with the LOBIN platform.

This paper describes in detail the LOBIN platform: a novel healthcare IT platform that allows monitoring several physiological parameters, such as ECG, heart rate, angle of inclination and activity index (movement), and body temperature, and tracking the location of a group of patients within hospital environments. The physiological information is measured using a wearable smart shirt based on e-textile and is transmitted through a low-cost WSN infrastructure owned by the hospital to the Management Server. Several tests have been performed in order to determine the maximum number of patients that the system is able to support providing a given degree of QoS and to generate design criteria for real deployments. Interesting results have been obtained that show that different WSN topologies have to be used depending on the target application. The LOBIN platform has been validated in a real scenario by setting up a pilot scheme at the Cardiology Unit of La Paz Hospital, Madrid, Spain, one of the leading hospitals in Spain.

The remainder of the paper is structured as follows. Section II introduces overall system requirements and architecture design as well as the main characteristics of each LOBIN subsystem. Section III describes system validation and testing. Finally, Section IV outlines the main conclusions of this study.

\section{LOBIN ARCHITECTURE AND SUBSYSTEM DESIGN}

The LOBIN system is defined as a healthcare IT platform designed to meet the following requirements.
1) Both to monitor several physiological parameters and to track the location of a group of patients within hospital facilities.

2) The physiological parameters to be monitored are: ECG, heart rate, angle of inclination, activity index, and body temperature.

3) The device used to provide such information must be wearable, noninvasive, comfortable, and washable.

4) All the physiological data associated with a patient must be stored for some period of time.

5) It must be possible to visualize ECG on-demand in real time.

6) The location algorithm must be accurate enough to determine correctly the hospital room where a given patient is actually located.

7) The system must allow configuring alarms by setting different triggers associated with each patient.

The system must also allow both monitoring, in a noninvasive manner, critical physiological parameters from patients who suffer high-risk diseases and have very low mobility (e.g., ECG from patients suffering diseases of the heart and blood vessels and who, usually, are confined to a bed) and obtaining other ordinary parameters from patients whose health is not so weak and have medium mobility (e.g., temperature monitoring of patients in a hospital).

Furthermore, the system must support the transmission of certain parameters when the Management System explicitly requests them (on-demand) or after any alarm occurs.

Fig. 1 shows the overall architecture designed to fulfil these requirements, which consists mainly of the following four subsystems.

1) The healthcare-monitoring subsystem consists of the set of smart shirts to be worn by the patients. Each smart shirt is provided with a device (namely wearable data-acquisition device (WDAD)) to collect and process the physiological parameters and to transmit them wirelessly.

2) The location subsystem consists of a beacon point (BP) infrastructure, which signals well-known positions, and a 


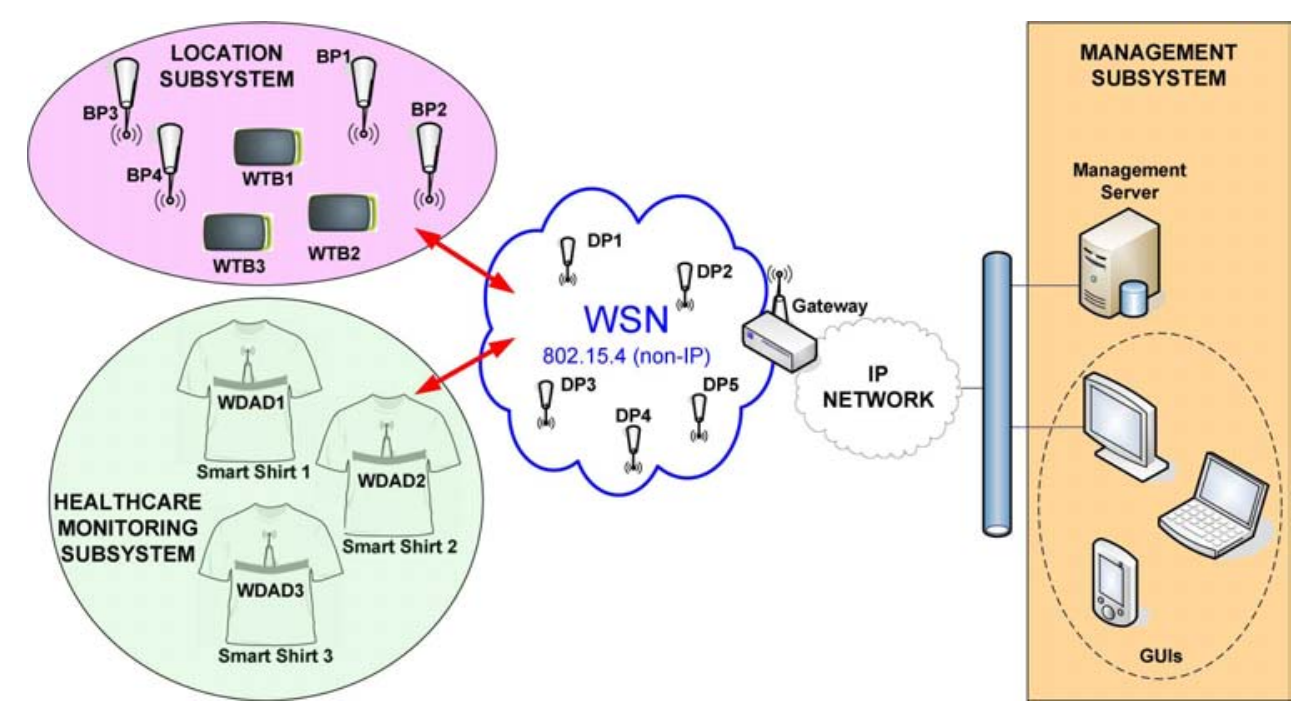

Fig. 1. System architecture.

set of end devices (namely wireless transmission boards (WTBs)) to be carried by targeted users (e.g., patients or any other personnel from the hospital). The BPs are deployed in well-known positions and send messages periodically to aid location. End devices (WTBs) collect signal strength information received from different BPs and send it wirelessly. For the sake of flexibility and versatility, the healthcare-monitoring subsystem and the location subsystem are completely hardware independent. Both the healthcare-monitoring parameters and the location information are transmitted and delivered to the management subsystem by the WSN subsystem.

3) The WSN subsystem consists, in general, of a set of devices (namely distribution points (DPs)), which transmit data ad hoc via a gateway that forwards them to the management subsystem. Therefore, the gateway consists of two different printed-circuit boards (PCBs): one to interface with the WSN infrastructure (gateway WTB) and one to interface with the wired infrastructure (gateway Ethernet transmission board (ETB)).

4) The management subsystem represents the IT infrastructure that handles the information associated with every single patient. It consists of a Management Server that processes and stores these data and graphical user interfaces (GUIs) for the hospital staff to monitor the status of the patients. This subsystem could be integrated into hospital patient information management systems.

Table II summarizes the hardware used to implement the LOBIN devices.

\section{A. Healthcare-Monitoring Subsystem}

Over the past few years, monitoring systems based on multifunctional instrumented garments have been playing an innovative role in the development of more human-oriented monitoring devices. These biomonitoring systems have recently evolved considerably due to the appearance of smart fabrics. The technology of smart fabrics allows adding functionalities to tex-
TABLE II

HARDWARE SUMMARY

\begin{tabular}{cl}
\hline \hline Device & \multicolumn{1}{c}{ Hardware Description } \\
\hline Healthcare WTB & MC13213 (Freescale Semiconductor) \\
Location WTB & $\mu$ processor: 689S08A (8 bit architecture) \\
Beacon Point & Transceiver: MC1320x (802.15.4 compliant) \\
Distribution Point & Flash memory: $60 \mathrm{~KB}$ \\
Gateway WTB & RAM: 4 KB \\
& RCM3720 (Rabbit Semiconductor) \\
& $\mu$ processor: Rabbit3000 (8 bit architecture) \\
Gateway ETB & Clock frequency: $22 \mathrm{MHz}$ \\
& Flash memory: $512 \mathrm{~KB}$ \\
& SRAM: $256 \mathrm{~KB}$ \\
\hline \hline
\end{tabular}

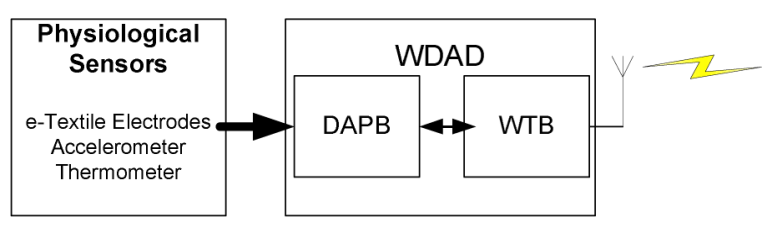

Fig. 2. Healthcare-monitoring subsystem block diagram.

tiles. This innovative technology is based on the combination of known materials to obtain major benefits. The original idea comes from the study of biological systems, where organic and inorganic materials are combined in an effective way. Smart fabrics of special interest to this work are the conductive fabrics, which combines conductive materials (either metallic or nonmetallic) with organic textiles such as nylon or lycra (e-textiles).

Conductive fabrics allow a comfortable and user friendly way to monitor patient's health status over extended periods of time in many different fields, such as biomonitoring, telemedicine, home healthcare, rehabilitation, or sport medicine [11], [12].

The LOBIN healthcare-monitoring subsystem is mainly based on conductive fabrics and e-textile. It consists of a set of physiological sensors and a WDAD, which processes the data coming from the sensors and transmits them wirelessly. The WDAD is further divided into two different PCBs: the data acquisition and processing board (DAPB) and the WTB. Fig. 2 illustrates how the healthcare-monitoring subsystem works. 


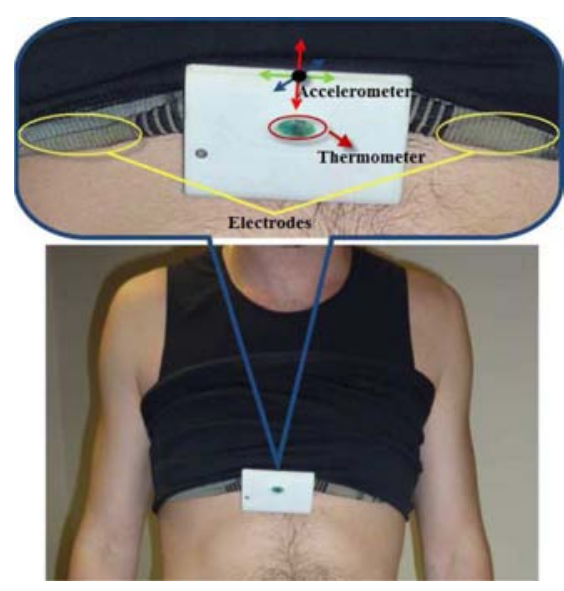

Fig. 3. Physiological sensors.

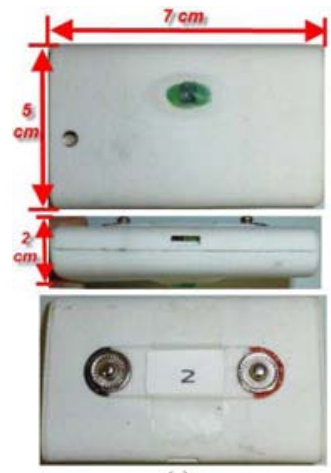

(a)

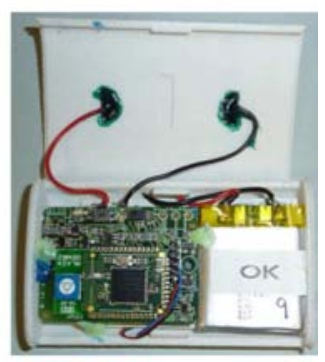

(b)
Fig. 4. (a) WDAD. (b) Healthcare-monitoring WTB.

The physiological sensors are in charge of measuring raw data that will be further processed in order to obtain the required biomedical parameters. The available sensors, as shown in Fig. 3, are as follows.

1) An e-textile-based tape that measures the bioelectric potential of the human body. Its most relevant feature is the integration of two e-textile electrodes to perform the biomedical data acquisition. This e-textile-based tape is integrated into a wearable, noninvasive, comfortable, and washable smart shirt.

2) A three-axis accelerometer that detects the movements of the device in the three axis. It is integrated into the WDAD and provides a way to detect patient movements and to determine if the patient is laying down or moving about in order to aid appropriate diagnosis of received signals.

3) A thermometer that measures the body temperature. It is also integrated into the WDAD and must be in direct contact with the skin of the patient.

Fig. 4(a) shows the WDAD developed under the LOBIN project. The WDAD is composed of the DAPB and the WTB, which is shown in Fig. 4(b).

The DAPB collects all the data from the sensors, processes them, merges them all together in a message and sends them via a serial port to the WTB. Fig. 5 illustrates how the DAPB works.

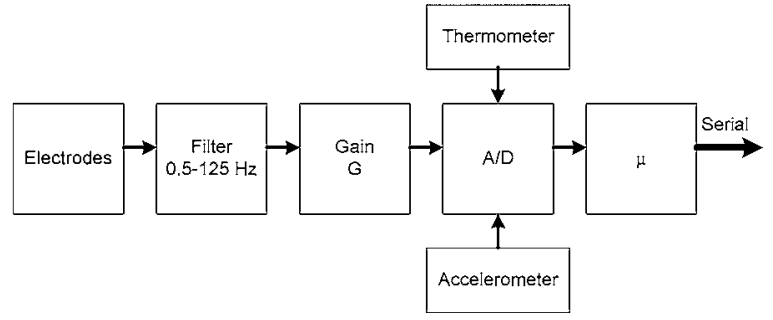

Fig. 5. DAPB block diagram.

The WTB builds a new packet by adding information related to the WSN to the message coming from the DAPB and transmits it wirelessly to the management subsystem. The WTB consumes $20 \mathrm{~mA}$ in standby mode, $30 \mathrm{~mA}$ when transmitting, and $37 \mathrm{~mA}$ when receiving. Both the DAPB and the WTB share the same battery and in a future commercial phase could be integrated onto a common PCB. During the evaluation phase and using a $600 \mathrm{mAh}$ battery, as shown in Fig. 4(b), the lifetime was about 8-9 h (see Section III-D).

Both the physiological sensors and the WDAD are integrated into a wearable, noninvasive, comfortable, and washable smart shirt from Textronics, Inc. [13], which is shown in Fig. 3.

As has already been mentioned, a healthcare-monitoring frame to packetize all the sensitive information in just one message is defined. All the physiological parameters are sampled every $4 \mathrm{~ms}$. However, this message is only transmitted after collecting 65 ECG samples. This value is determined by the size of the frame resulting from the healthcare-monitoring frame together with the additional WSN information, needing to be as close as possible to the 802.15.4 MTU (102 B). As a result of this decision, efficiency is maximized and transmission rates decreased, which, in turn, reduces collisions in the WSN. Thus, from the management subsystem point of view, the rest of the parameters apart from the ECG are sampled every $260 \mathrm{~ms}$.

Table III summarizes the most important features of the parameters transmitted in this message.

\section{B. Location Subsystem}

Nowadays, user location is a hot topic for Industry since there are many location-based services (LBSs) that can provide added value to different kinds of applications.

In the state of the art, there are many different methods [14], [15] to compute the location of a user, such as cell global identity (used in wireless local area network (WLAN)/global system for mobile communication (GSM)), angle of arrival (AoA), received signal strength (RSS), time of arrival (ToA), time difference of arrival (TDoA), or enhanced observed time difference (E-OTD). The suitability of each method depends on whether the location is outdoors or indoors and on the technology used.

Regarding indoor location, much research has been carried out recently and many different solutions using different technologies, such as assisted-GPS (AGPS) [16], 802.11 [17], Bluetooth [18]-[20], radio frequency identification (RFID) [21], [22], ultra wideband (UWB) [23], or 802.15.4/Zigbee [8], have been explored. 
TABLE III

HeALThCARE-MONITORING SUbSySTEM PARAmETERS

\begin{tabular}{|c|c|}
\hline Parameter & Features \\
\hline ECG & $\begin{array}{c}\text { Frequency: } 0.5-125 \mathrm{~Hz} \\
\text { Amplitude: } \sim 0-50 \mathrm{mV} \\
\text { Gain: } \mathrm{G} \\
1 \text { byte/ sample } \\
65 \text { samples/tx packet }\end{array}$ \\
\hline Heart rate & $\begin{array}{c}\text { Computed from ECG } \\
1 \text { byte/sample } \\
1 \text { sample/tx packet }\end{array}$ \\
\hline Angle of inclination & $\begin{array}{l}\text { Computed from 3-axis } \\
\text { accelerometer } \\
1 \text { byte/sample } \\
\text { I sample/tx packet }\end{array}$ \\
\hline Activity index & $\begin{array}{c}\text { Computed by averaging angle of } \\
\text { inclination } \\
1 \text { byte/sample } \\
\text { I sample/tx packet }\end{array}$ \\
\hline Body temperature & $\begin{array}{c}\text { Range: }-20^{\circ} \mathrm{C} \text { to } 120^{\circ} \mathrm{C} \\
\text { Accuracy: } 0.1{ }^{\circ} \mathrm{C} \\
\text { Valid range: } 32^{\circ} \mathrm{C} \text { to } 45^{\circ} \mathrm{C} \\
1 \text { byte/sample } \\
1 \text { sample/tx packet }\end{array}$ \\
\hline Level of battery & $\begin{array}{c}100-0 \% \\
\text { Coverage: } 8-9 \text { hours } \\
1 \text { byte/sample } \\
1 \text { sample/tx packet }\end{array}$ \\
\hline Alert & $\begin{array}{c}\text { To carry alert code if necessary } \\
1 \text { byte/sample } \\
1 \text { sample/tx packet }\end{array}$ \\
\hline
\end{tabular}

The use of each technology implies some benefits and drawbacks that make it more or less suitable depending on the targeted scenario.

During the LOBIN location subsystem development phase, the performance of three different indoor location algorithms was compared in real environments.

1) Algorithm based on triangulation: Deriving the distance from the RSS is tried both by means of the Friis formula and by using regressions based on empirical measurements taken within the target indoor scenario. In both cases, the obtained results do not fit reality and the users' actual locations are not determined accurately. Therefore, this location algorithm is ruled out.

2) Algorithm that does not use the link quality information (LQI) to determine the user's actual position: This algorithm is very simple. The location is computed as the geometrical center of the BPs within the coverage of the end node to be located. This algorithm locates the user always within the area comprised by the appropriate BPs, but it offers very poor precision, which, in turn, depends strongly on the distance between BPs and on the transmitted power. Therefore, this location algorithm is also ruled out.

3) Algorithm based on weighted centroid localization (WCL) and the LQI: The WCL algorithm [24] consists of computing a point comprised within the area covered by the BPs the target end node received beacons from, so that this point will be closer to those BPs from where higher LQIs are received. In order to do so, different weights (directly proportional to the received LQI) are associated to each received beacon. This algorithm is the one used in the LOBIN platform since it yields the best results.

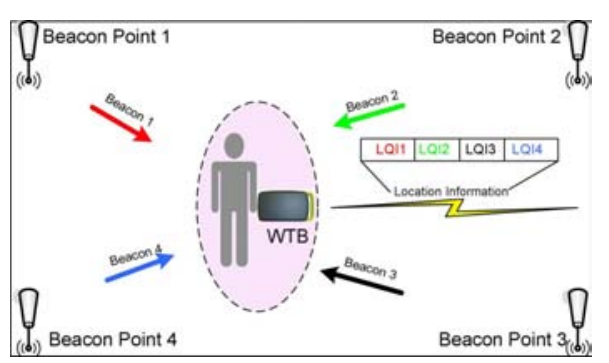

Fig. 6. Overall location subsystem architecture.

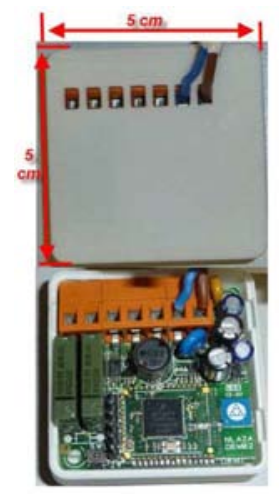

(a)

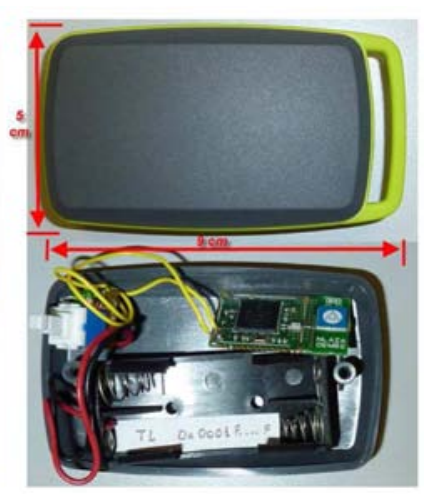

(b)
Fig. 7. (a) BP. (b) Location WTB.

The LOBIN location subsystem consists mainly of two different devices: BPs and WTBs. Fig. 6 sketches how the location subsystem works.

The BPs are deployed in well-known positions and are plugged into the electricity supply network. They send beacons periodically in one of four available channels using a fixed and well-known transmission power. Fig. 7(a) shows such a device.

The WTBs are carried by the users and are in sleep mode most of the time. Periodically (namely every $9 \mathrm{~s}$ ), they wake up and listen to every single channel available for location purposes in order to receive beacons from the BPs. To be precise, they record the LQI received from the different BPs. Note that the time that the WTBs are listening to a given channel has to be at least twice the time between beacons from a given BP in order to avoid beacon loss. Once these data are collected, the WTBs merge them into a single message, add some necessary network-layer information, and send it to the management subsystem through the WSN communication infrastructure. The hardware used to develop this PCB is exactly the same as the one used for the WTB from the healthcare-monitoring subsystem. However, the location subsystem WTBs are powered with two AAA batteries. The battery lifetime was tested to be about two days for the chosen configuration (see Section III-B). Fig. 7(b) shows the location WTB.

With all the collected data, the WTB builds the location subsystem frame. Such a frame consists basically of a set of pairs [BP identifier, received LQI]. This set is preceded by a field that identifies the number of transmitted pairs up to a maximum of 80, which is enough for the application requirements. Since the position of the BPs is well known by the management sub- 
system, the location of the patients within a 2-D plane can be computed and then drawn onto a map.

The LOBIN location algorithm can support the following features.

1) The patient is always located within the area covered by the BP infrastructure, e.g., a hospital.

2) The accuracy of the algorithm varies from one location decision to another mainly because of multipath issues.

3) The accuracy of the location algorithm could be enhanced by increasing the density of BPs. However, this would increase the deployment cost of the BP infrastructure. Therefore, a tradeoff solution was chosen (see Section III-B), taking into account the location requirements of this application, i.e., it must be accurate enough to determine correctly the hospital room where a given patient was actually located.

4) For the selected configuration, the location latency was $9 \mathrm{~s}$, since the WTBs wake up, listen to the different available channels for beacons, and send the collected data within this period. If this parameter was reduced, the latency would be lower, but it would imply higher battery consumption. Therefore, this value also represents a tradeoff between both parameters taking into consideration the low mobility requirements for hospital environments.

\section{WSN Subsystem}

WSNs are small networks that require low bandwidth, low power consumption, and low deployment and maintenance costs. Recently, they have become increasingly important in the telecommunication industry because of their wide range of applications.

In order to promote and encourage the incorporation of such networks in a competitive market, the IEEE has defined the 802.15 family of standards that deal with the physical and media access control (MAC) layers. Within this family of standards, it is especially relevant to the work presented in this paper the Low-Rate Wireless Personal Area Networks IEEE 802.15.4 [25]-[27], that was designed for applications with low transmission rate, very low power consumption, and relaxed QoS requirements.

Although the Zigbee Alliance addresses the upper layers (namely network and application) and proposes several routing algorithms to be used at the network layer, over the past few years, much research has been carried out in order to design and develop routing algorithms that fit within the many restrictions of such networks in an effective way [28]-[31]. These restrictions have to do mainly with the limitations of the available hardware (e.g., battery, memory, computing capacity) and with some other issues associated with wireless communications such as scarce bandwidth or collisions.

The targeted scenario for the LOBIN project adds some additional complexity to the problem. Such a scenario presents an asymmetric traffic pattern. Most of the information is sent from the patients to the management subsystem (uplink), since patients are sources of data, whereas only a few commands travel in the other direction, i.e., from the management subsystem to

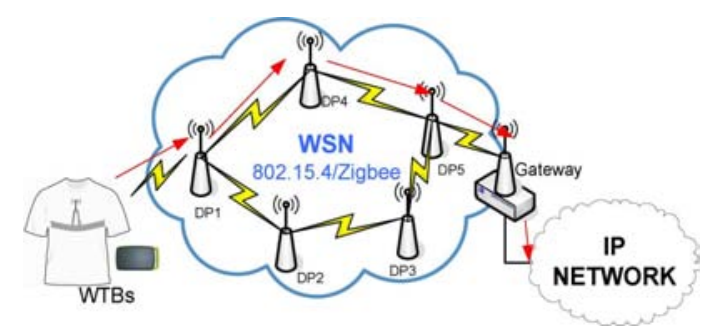

Fig. 8. Overall WSN subsystem architecture.

the patients (downlink). As a result, a routing algorithm over 802.15.4 has been designed and developed within the LOBIN project in order to fit the special features of the system.

The LOBIN WSN subsystem consists of three different devices: WTB, DP, and gateway. Fig. 8 shows the overall WSN subsystem architecture.

The WTBs have been already presented. They are the devices that are in charge of transmitting wirelessly both the healthcare monitoring and location data.

DPs are deployed all over the targeted areas. They transmit either healthcare-monitoring data or location data ad hoc via a gateway that forwards them to the management subsystem. They are plugged into the electricity supply network. From the hardware point of view, they are exactly the same as the BPs, shown in Fig. 7(a). The only difference being the software that runs on them.

Gateways are devices that incorporate a wireless interface and an Ethernet (IEEE 802.3) interface. They are in charge of forwarding all the data coming from the WSN to the management subsystem. They are plugged into the electricity supply network. Like DPs, they implement full routing functionality. Moreover, end nodes can send data straight through them.

A gateway consists of two different PCBs: the WTB and the ETB. The gateway WTB is based on the same chip as any other WTB (namely, MC13213 from Freescale Semiconductor), but it is deployed on a PCB that supports connectivity to additional interfaces like Ethernet, GSM, etc. The gateway ETB is based on the RCM3720 PCB from Rabbit Semiconductor and it is equipped with an Ethernet interface. The communications between both PCBs is performed via a serial port at $115600 \mathrm{Bd}$. This value has been set for the gateway to be able to forward, without problems, the aggregate traffic coming from the WSN. Fig. 9(a) illustrates how the gateway works and Fig. 9(b) shows the gateway itself.

The routing algorithm used in project LOBIN is a source routing algorithm based on dynamic source routing (DSR) [32]. When a DP is turned on, it queries its neighbor DPs to discover how to reach the destination (i.e., the gateway). Thereafter, it receives responses, which contain different paths to do so. The first received response is stored as the default route. Following responses (up to 2) are stored as back-up routes. Moreover, DPs exchange, periodically, status information with their neighbors. Nevertheless, since the targeted scenario is very stable, this period is set to a high value (namely, minutes) in order to reduce the overhead introduced into the WSN. Therefore, if a DP realizes that the default path is down either while transmitting a 


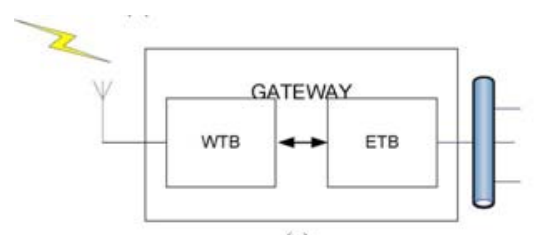

(a)

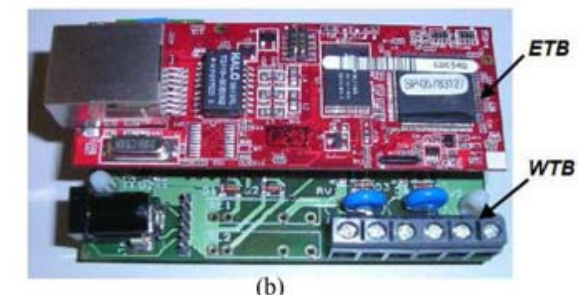

(b)

Fig. 9. (a) Gateway block diagram. (b) Gateway.

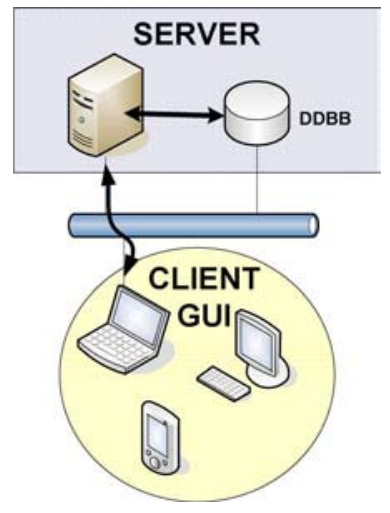

Fig. 10. Management subsystem client-server architecture.

packet or after exchanging routing information, it can use one of the default paths to immediately solve the problem. If there is no back-up path available in the DP at any given moment, the path discovery procedure is triggered again. Some additional checking is performed in each DP to avoid loops.

In order to save memory in the DPs, they are not aware of the end nodes (i.e., healthcare-monitoring WTB or location WTB) that are associated to them. However, end nodes do store the DP they are associated with. If, either because the end node roams from one DP to another or because the DP it is associated with goes down, the end node realizes its DP is no longer available and it looks for other DPs to send the data through. Furthermore, in order to save battery life, end nodes are not involved in routing.

\section{Management Subsystem}

The management subsystem consists of a Management Server that processes and stores data associated with the patients and GUIs for the hospital staff to monitor the status of the patients. The management subsystem is based on a client-server architecture, as shown in Fig. 10.

The server was developed using $\mathrm{C}$ as the programming language. It runs on Fedora, which is an operating system built on top of the Linux kernel. It uses an Oracle database (DDBB) to store the information associated with the patients. The database provides an independent interface with the GUI and allows the development of tailored user applications to cover additional functionalities.

The client was developed in Java, which provides it with great flexibility and allows it to run on any platform without problems, either a computer, a PDA or a mobile.

The Management System must offer a simple and intuitive interface where it integrates the functionalities of biomonitoring and location of numerous patients. The requirements that these interfaces must satisfy are listed as follows.

1) To manage the patients, i.e., to add, modify, or delete patient profiles.

2) To monitor in real time, all the medical parameters of any patient.

3) To visualize the file with all the measured values of the healthcare-monitoring subsystem parameters over a period of time.

4) To locate any patient within the space qualified for its stay.

5) To verify if any alarm has been activated. Additionally, this information can be sent by short message service (SMS).

6) To generate forms with all the data of the patient and the measured medical parameters.

7) To allow setting whether a patient must constantly send information in real time or upon demand.

8) To request information of any medical parameter of a patient upon demand.

In health environments, the privacy of the patients is very important. Therefore, it is mandatory for the system to require authentication of users, which entails services for user administration and permissions.

For this application to be implemented in any hospital without the necessity to modify the code of the location module, it is required to provide tools for managing maps, end nodes (Location WTBs), and BP locations.

\section{SYSTEM VALIDATION AND TEST}

First, each subsystem has been tested separately in order to check the correct integration of the different subsystems in a laboratory environment without real patients. The main goal of this first stage was to test the correct collection, transmission and processing of data by the LOBIN platform using traffic pattern generators. Next, the overall system was tested in a real scenario (namely, as a pilot scheme deployed in the Cardiology Unit of La Paz Hospital).

Management subsystem tests are not included separately. However, typical software development tests have been applied to this system in order to check its behavior and performance during the development phase. Furthermore, usability tests have been performed during the last stage of the validation process (i.e., tests in a real scenario).

\section{A. Healthcare-Monitoring Subsystem Tests}

The performance of the healthcare-monitoring subsystem has been checked with a set of laboratory tests. These tests can be further divided into two different subsets. 


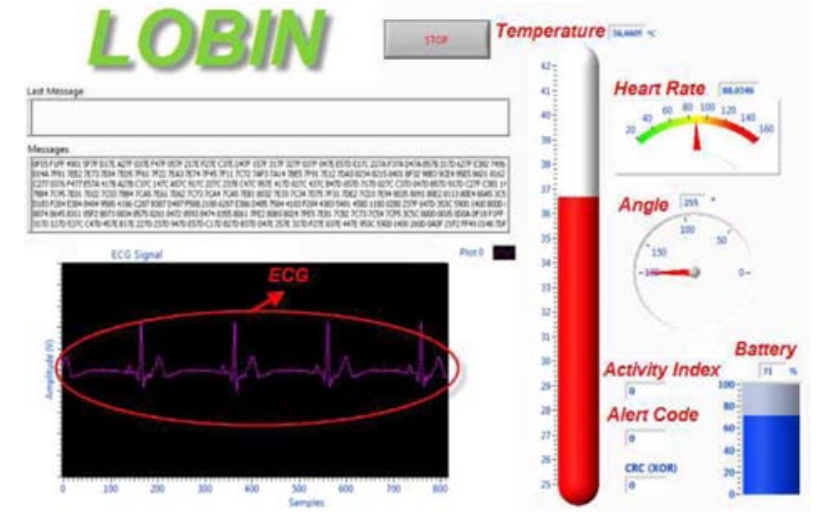

Fig. 11. Test interface.

The first subset of tests tries to verify that the sensors collect real data and that the WDAD processes and transmits these data correctly.

In order to verify the correct processing of the bioelectric potentials obtained by the e-textile electrodes, the mensurations sent by the WDAD are compared with the real mensurations generated in the laboratory. For the generation of the bioelectric potential, we have used a National Instruments generator NI USB 628, managed by software using LabVIEW. In addition to simulating normal ECG signals, we have also tested different arrhythmias with the aim of verifying the operation of the alarms.

To verify that the body temperature sensor works properly, its mensurations have been compared with the mensurations of a commercial thermometer. It has been determined that the temperature measured by the thermometer integrated into the WDAD takes about $5 \mathrm{~min}$ to acquire an accurate value.

The accelerometer tests have been performed manually by tilting the device to known angles.

Fig. 11 shows the interface that has been developed for this first validation stage using LabVIEW in order to verify all the results and to provide information on all selected biometrical parameters: ECG, heart rate, angle of inclination, activity index, temperature, battery, etc.

The ECG representation allows perfectly identifying the waves and intervals of the heartbeat, consisting of a primary (P) wave, a QRS complex and a T wave, as shown in Fig. 12.

The second subset of tests verifies that the subsystem works in an environment closer to the actual target. The main goal of this stage was to check qualitatively that the sensors work properly when in direct contact with a human body.

The tests have been performed by using both a standalone e-textile belt and one that was integrated into a shirt. When the patients were not carrying out activities that required very vigorous movements, both e-textile articles yielded good results. However, during sudden movements such as standing up or jumping about, both results worsened slightly. Even so, the electrodes integrated in the shirt performed better in such situations since the shirt helps to maintain sensor contact with the user's chest. If some conductive substance (e.g., water or conductive gel) was applied to the electrodes, the effects of sudden

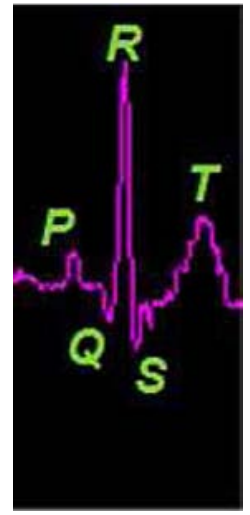

Fig. 12. ECG with waves tags.

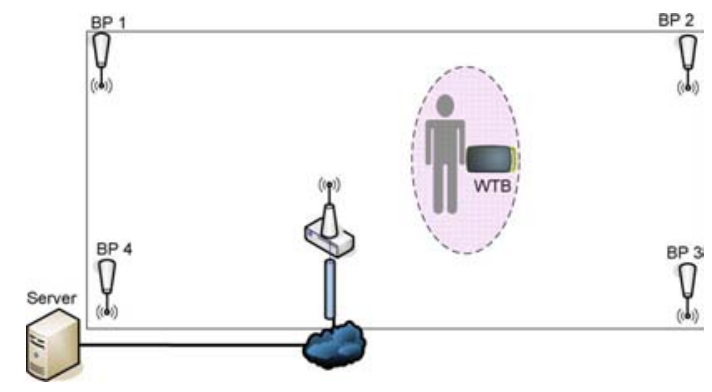

Fig. 13. BP density tests.

movements to the quality of the ECG signals were, in both cases, mitigated.

Taking into account these results, we concluded that they were satisfactory and that the healthcare-monitoring subsystem provided a means to capture biometrical parameters in a noninvasive manner. In order to avoid reducing the accuracy of the measured ECG signal for patients who are not usually bedridden, then, according to our results, the use of a conductive substance is a good practice.

\section{B. Location Subsystem Tests}

The objective of these tests was to verify that the location algorithm developed within the scope of the LOBIN project worked in real scenarios as well as to select the most appropriate values for the parameters and to study their impact on the performance of the algorithm. Thus, the location subsystem tests can be divided into two different subsets.

The main goal of the first subset of tests was to determine how the number of BPs and the distances between them impact the accuracy of the location algorithm.

In order to be able to locate end nodes (WTBs) correctly, it was necessary to deploy a rectangular grid of BPs. Only the end nodes (WTBs) that were within this grid would be located accurately.

Initially, the end nodes were tried to be located within rectangular areas of variable size bounded by BPs. Fig. 13 sketches such a scenario.

Several tests were performed over areas of $100(10 \mathrm{~m} \times 10 \mathrm{~m})$, $225(15 \mathrm{~m} \times 15 \mathrm{~m})$, and $400(20 \mathrm{~m} \times 20 \mathrm{~m}) \mathrm{m}^{2}$. The location 


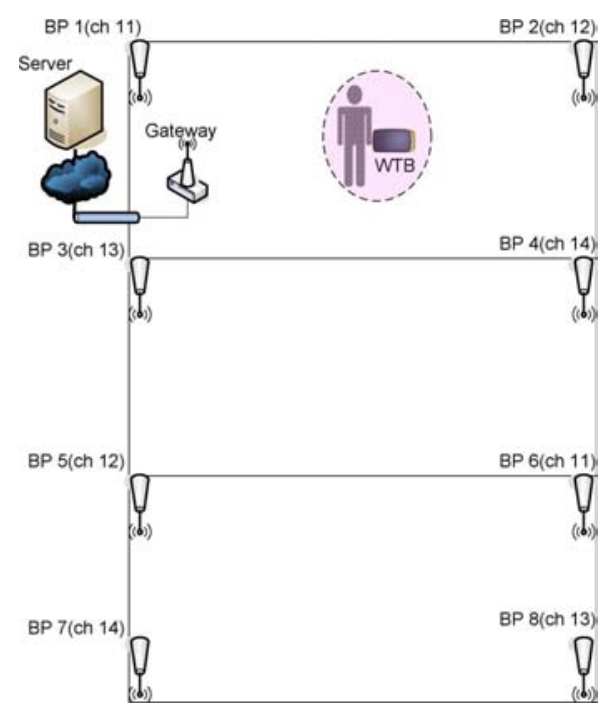

Fig. 14. Scalability test.

algorithm was checked to perform better for those deployments where the BPs were separated as much as their coverage range (i.e., $100 \mathrm{~m}^{2}$ and $225 \mathrm{~m}^{2}$, since the coverage range of such devices is between 10 and $15 \mathrm{~m}$ ). This result actually makes sense since for higher distances the end node may not receive beacons from some of the deployed BPs, and therefore, the location algorithm is run using less information. Therefore, it makes also sense that the closer the BPs are deployed the better, since the probability of missing a beacon is reduced. As a result, it was decided to deploy BPs at the edges of subrectangles of $100 \mathrm{~m}^{2}$ of area.

However, it is also necessary to locate users over wider areas. Hence, additional tests were carried out in order to verify whether the system still worked correctly when deploying a rectangular grid with more BPs. Following the design criteria mentioned in previous paragraph, the scenario shown in Fig. 14 was tested, where eight BPs were deployed to cover an area of $300 \mathrm{~m}^{2}$.

The channel assignment of each BP is not random. The four channels that are furthest from the channel used to send healthcare information (i.e., channel 25) are selected. Furthermore, for deployment, it is recommended to place the BPs operating on the same channel as far from each other as possible. These rules are not mandatory, since theoretically the channels in 802.15.4 are independent, but, in practice, its use is highly recommended to avoid possible interference, which may modify the beacons LQI.

The accuracy in the location of end nodes (WTBs) for the scenario shown in Fig. $14\left(300 \mathrm{~m}^{2}\right)$ was proved to be very similar to the accuracy obtained previously for the scenario of just $100 \mathrm{~m}^{2}$. This result shows that the system scales properly.

During this first subset of tests, it has been proven that the location subsystem performed properly for BP grid networks when BPs were deployed in subrectangles of $100 \mathrm{~m}^{2}$. Nevertheless, two problems were identified.
TABLE IV

TESTED CONFIGURATIONS

\begin{tabular}{cccc}
\hline \hline Test & Ptx_loc (s) & Tlisten (s) & Pbeacons (s) \\
\hline 1 & 30 & 2 & 1 \\
2 & 9 & 2 & 1 \\
3 & 20 & 4 & 1 \\
4 & 15 & 2 & 0.5 \\
5 & 9 & 2 & 0.5 \\
\hline \hline
\end{tabular}

1) Instability: After correctly being located on the map, an end node's (WTB's) position starts oscillating, without any actual movement by the end node.

2) Refresh time: When an end node (WTB) moves from one position to another, its new position takes time to be realized.

The instability problem can be due to loss of beacon packets, which may generate incorrect calculations for the end node's position.

The refresh problem was directly related to the period of time WTBs were sending location information with (Ptx_loc). The quicker the patient's location was updated, the earlier were patient movements detected. However, WTBs then consume more battery power. In order to keep battery power under control, the period of time WTBs sent location information with (Ptx_loc) was set to be equal to or greater than $9 \mathrm{~s}$. This location update time represents a tradeoff between battery power consumption and location latency that meets the application requirements (i.e., low/medium mobility).

The time periods between WTBs sending location information with (Ptx_loc) is conditioned by following other parameters.

1) The period between BPs sending beacons (Pbeacons).

2) The time end nodes spend listening to each available location channel (Tlisten).

3) The number of channels available (Nchannels).

Ptx_loc must be always higher than the time the end node needs to listen to all the channels available for location.

$$
\text { Ptx_loc } \geq \text { Tlisten } \cdot \text { Nchannels. }
$$

Also, the time end nodes spend listening to each channel (Tlisten) must be at least twice the period of transmission of beacons in order to ensure there is enough time to receive at least one beacon.

\section{Tlisten $\geq 2 \cdot$ Pbeacons}

It is also necessary to obtain location information from 4 BPs for the location mechanism to work properly.

Taking all these restrictions into account, a second subset of tests was designed. The main goal of this second subset of tests was to explore what are the most appropriate values for all these parameters, i.e., the configuration that yields better performance, reducing as much as possible the effects of the two problems detected in the first subset of tests (i.e., instability and refresh time). In order to do so, five tests were performed selecting different values for Ptx_loc, Tlisten, and Pbeacon for each test. Table IV shows a summary of the different test configurations. 


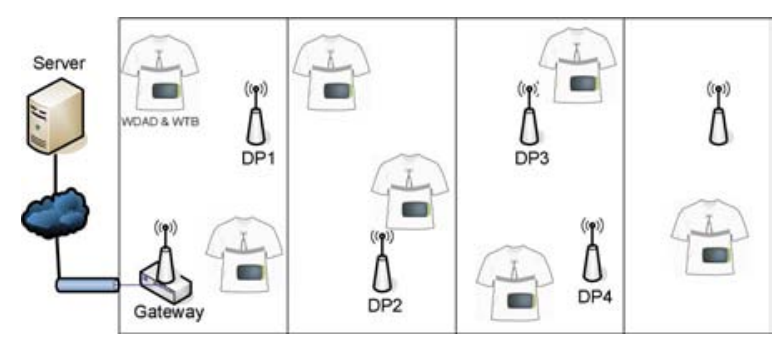

Fig. 15. DP network test.

It was checked qualitatively that the configuration set in test number 5 performed better than the others, so it was the one chosen for the deployment of the LOBIN platform in real scenarios, such as the pilot scheme in the Cardiology Unit at La Paz Hospital.

In this configuration, Ptx_loc was set to the minimum value in order to reduce the latency and to improve the location of patients when they were moving around. To reduce the probability of losing a beacon, the time for listening to each channel available for location (Tlisten) was set to four times the period of beacon transmissions (Pbeacons). Hence, if a beacon was lost there was still enough time to receive at least another two. This decision improved significantly the stability of the location algorithm.

In summary, performed tests have shown that the accuracy of the indoor location algorithm meets the system requirements. The margin of error was about $2 \mathrm{~m}^{2}$. This error margin improves the results obtained in [24], even despite of the fact that the results presented in [24] were obtained outdoors. This is mainly because de BPs are placed much closer one to another in the LOBIN platform than in the experiments carried out in [24] $(10 \mathrm{~m} \times 10 \mathrm{~m}$ in the LOBIN platform and $43 \mathrm{~m} \times 43 \mathrm{~m}$ in [24]).

The stability was also good enough with a moving patient, although there was a 9-s gap between refreshes, which was acceptable since, in hospital environments, the location in strict real time is not necessary due to the low to medium user mobility.

\section{WSN Subsystem Tests}

These tests tried to verify that the WSN satisfies the requirements of the system. This WSN has very specific requirements; i.e., it must support a minimum of five end nodes (WTBs) and five WDADs, and must offer a QoS that ensures that the percentage of lost packets is not more than $2 \%$ of those sent.

There are several possible network deployments. The most suitable for our system was determined by its behavior in these tests.

The first test deployment was composed of a network of distributions points (DPs) and a gateway. In order to cover approximately $300 \mathrm{~m}^{2}$, consisting of several laboratories, five DPs, and one gateway were deployed, as shown in Fig. 15.

Fig. 16 illustrates how packet loss increases when the traffic coming from the biomonitoring terminals increases. It can be checked that the percentage of lost packets increases nonlinearly with the number of devices and greatly exceeds the maximum loss percentage allowed (2\%).

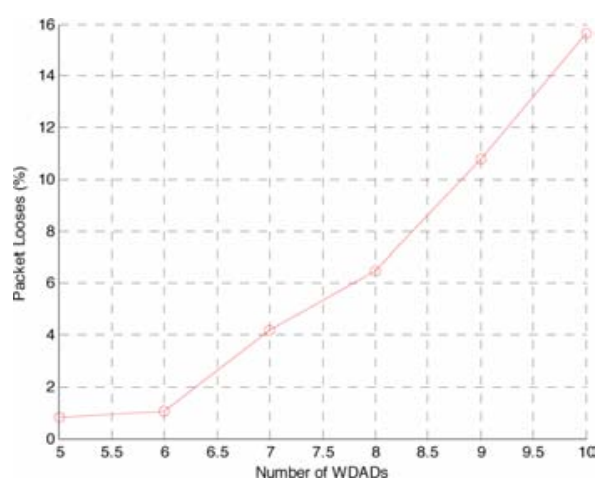

Fig. 16. DP network test results.

This large increase in losses was due to an almost exponential increase in traffic, as not only increases the traffic added by the sources, but also the traffic forwarded by the DPs. Similar issues were also found and reported in [8].

The system bottleneck was determined to be located in the network of DPs (WSN). This network was able to support many location WTBs, offering low traffic to the network, but was not able to support more than six WDADs sending messages every $260 \mathrm{~ms}(\sim 3.1(\mathrm{~kb} / \mathrm{s}) / \mathrm{WDAD})$.

To solve this problem, the solution using two different channels within the WSN in order to split the traffic was explored at first. Therefore, the infrastructure of DPs and gateways were duplicated and half of the devices were reprogrammed so that they worked over another 802.15 .4 channel. It was tested with several of the 16 channels offered by 802.15 .4 and none of the test results yielded the expected improvement. Thus, it was concluded that in scenarios with heavy traffic, interference between 802.15.4 channels may occur with subsequent high data losses.

Interesting results and conclusions on the effects of interference on the performance of well-known systems working in the license-free ISM band, such as 802.11, 802.15.1 (Bluetooth), or 802.15.4, are presented in [33].Channel 25 has been chosen as default channel for the LOBIN WSN communication infrastructure taking these results into account, since they point out that this channel can be used for operation clear of 802.11 interference.

As an alternative to the former topologies, the network of DPs was replaced with several gateways connected directly to the hospital IP-network. Fig. 17 shows the new network configuration.

This solution provided the best results. It gave the lowest percentage of losses in scenarios with 8/10 matched WDADs and location WTBs. This percentage of looses was tested to be with a $95 \%$ confidence in the interval $(0.2068 \%, 1.6585 \%)$ for ten tests of about 15 min duration involving ten users. Therefore, the obtained data were considered to fit a Gaussian distribution and the $95 \%$ confidence interval was computed using the following formula, with $\mu$ being the mean of the data, $\sigma$ the standard deviation of the data, and $n$ the length of the data (i.e., $n=100$ ):

$$
\left(\mu-1.96 \frac{\sigma}{\sqrt{n}}, \mu+1.96 \frac{\sigma}{\sqrt{n}}\right) .
$$




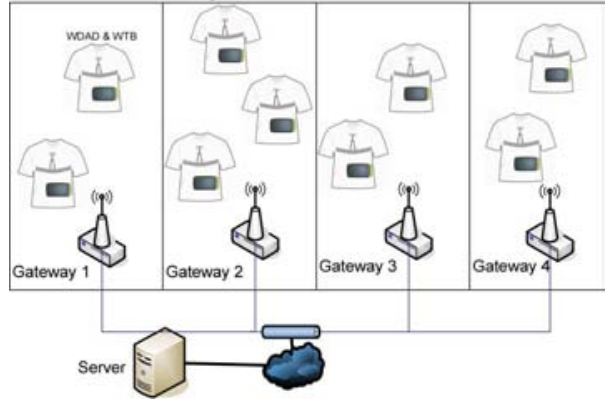

Fig. 17. Gateway network test.

This configuration also provides support for mobility, as both location and healthcare-monitoring information coming from a given device may be forwarded to the management subsystem by several gateways. Hence, if a WTB loses connectivity with a given gateway, there will be at least another available one to send this information to the Management Server. However, this configuration has the disadvantage that requires a wired infrastructure (namely Ethernet) to be deployed.

After completing these tests, it can be concluded that in order to monitor the ECG from several patients simultaneously, a network configuration with several gateways is more convenient. However, while monitoring other parameters such as temperature on demand or location applications with lower bandwidth consumption, a network of DPs provides fair enough results.

\section{Overall System Tests}

After tuning each subsystem separately, the performance of the whole system was tested in a real scenario by developing a pilot scheme in the Cardiology Unit at La Paz Hospital.

Fig. 18 illustrates the coverage area and network configuration infrastructure that was deployed for this pilot scheme.

Twenty-four-hour tests involving five patients were carried out. The main results from this test were considered successful because of the following reasons.

1) The percentage of packet losses was lower than the maximum acceptable value (i.e., 2\%). The percentage of looses was tested to be with a $95 \%$ confidence in the interval $(0.1875 \%, 1.5734 \%)$. It can be checked that such results even improve the results obtained during the laboratory tests (see Section III-C), which makes sense since the pilot scheme only involves five patients, whereas the laboratory tests involve ten patients.

2) Battery life was tested to be about $8-9 \mathrm{~h}$ for WDADs and two days for location WTBs.

3) Measured physiological parameters were normal and met expected values.

4) The quality of the real-time ECG signals was approved by hospital doctors. On the left-hand side, Fig. 19 shows the heart rate of five patients who were monitored, and on the right-hand side, it shows the real-time ECG for one of them.

The usability of the GUI was tested by hospital personnel with following feedback:

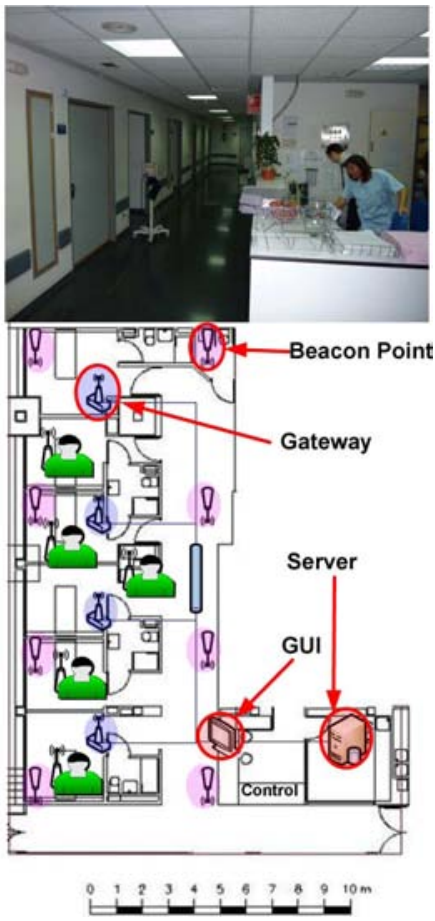

Fig. 18. Deployment of LOBIN platform in Cardiology Unit of La Paz Hospital.

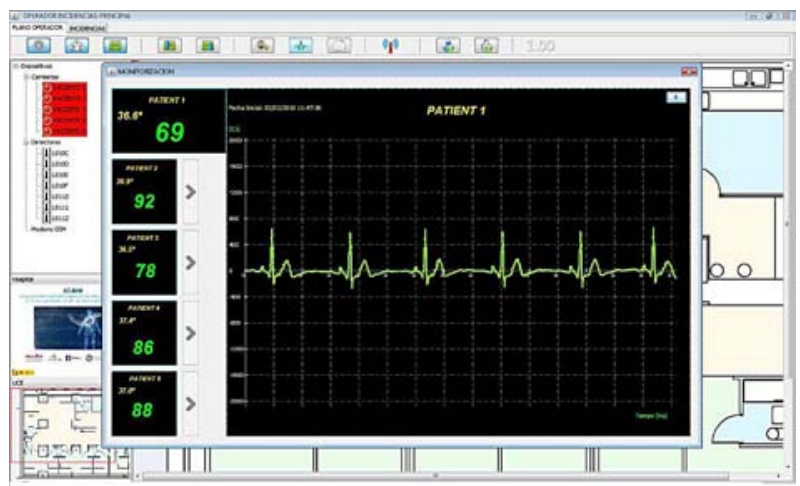

Fig. 19. Real-time ECG.

1) The tightness of the smart shirt was pointed out as an issue by hospital personnel, since patients from Cardiology Unit often present high abdominal perimeter. Nevertheless, no further complains were received.

2) Hospital staff use the GUI both to monitor the patients' status in real time easily, sitting in front of a PC, and to look at the signals over a long time period, taking advantage of the features provided by the tool in this sense. Some of these features, such as checking just a given period of time by selecting initial time and final time or the scroll, were incorporated in the GUI under request of hospital personnel.

3) The ECG display was modified following the feedback from the hospital personnel in order to fit the ECG representation they are used to handle (e.g., from commercial electrocardiographic devices). 


\section{CONCLUSION}

Throughout this paper, the main features of the hardware/software healthcare IT platform for hospital environments developed within the scope of the LOBIN project have been described. Such a platform provides a complete and versatile IT infrastructure, based on e-textile and WSN as most innovative technologies, both to monitor a set of physiological parameters from several patients and to locate them (or any other personnel from the hospital if desired) within hospital facilities. During the project's one and a half years duration, the LOBIN consortium has not only designed and developed this platform but also tested each subsystem separately and the overall system in a real environment, by deploying a pilot scheme in collaboration with the Cardiology Unit at La Paz Hospital. These results have been highlighted in this paper.

One of the key physiological parameters of the system is the ECG, which is measured using two e-textile electrodes integrated into a smart shirt. Currently, it is a major challenge to provide clinically required signal quality using this technology in any situation, because of motion artifacts or electrodes drying out. Along with the performed tests, it has been shown that the ECG signal quality is more than acceptable when patients stay still (i.e., normal conditions), but worsens when sudden movements take place (e.g., when patients get out of bed or walk around). Nevertheless, in the latter situations, the measured ECG signal is much more accurate if the electrodes are wet or covered with a conductive gel. In order to enhance the ECG signal quality in such situations, a mechanism to remove the motion artifacts, e.g., using the three-axis accelerometer information, is recommended [10].

The LOBIN platform also allows locating patients or any other hospital personnel provided with a location WTB. The developed indoor location algorithm is based on WCL and the LQI from 802.15.4/Zigbee. Its performance depends on several parameters such as the density of deployed BPs, the period BPs transmit beacons (Pbeacon), the time location WTBs spent listening to each channel available for location (Tlisten), and the period location WTBs send collected location data with (Ptx_loc). During the test phase, these parameters were tuned in order to achieve a tradeoff between accuracy, cost-effectiveness, latency, and battery consumption.

Reliability and capacity tests, in terms of maximum number of users, have also yielded interesting results. It has been checked that a DP network, where data are transmitted ad hoc between DPs until they reach the gateway, does not perform properly under heavy-traffic-load scenarios, such as five patients transmitting healthcare-monitoring information. For this kind of scenario, deploying a network of gateways has proven to be the most appropriate solution. This approach adds redundancy to the system and supports user mobility better, since WTBs may be within the coverage of different gateways and so data may be forwarded to the Management Server several times, reducing the probability of packet looses even when a patient is moving. Thus, the reliability of the system is improved.

However, it has been also checked that DP network infrastructures are suitable for applications that present low traffic load, but require high coverage, such as ordinary temperature checking on-demand or location fixing.

The LOBIN designed architecture, which decouples healthcare-monitoring and location subsystems, supports both scenarios. On the one hand, a network of gateways can be deployed to monitor in a noninvasive manner critical physiological parameters from patients who suffer high-risk diseases and present very low mobility (e.g., ECG from patients who suffer from disease of heart and blood vessels). On the other hand, a network of DPs can be deployed to monitor ordinary parameters, such as body temperature, either on-demand or periodically, from a large number of patients spread over a wide area (e.g., a hospital floor). In order to implement such an application within the LOBIN platform, few changes would be needed to the WDAD.

Furthermore, the DP network approach can allow deploying different LBSs seamlessly within the LOBIN platform, e.g., such a solution for the wireless segment of the system can be applied to support a service where the location of hospital staff is tracked in order to alert the appropriate person (e.g., doctor, nurse) who is closest about an eventual problem.

During the validation of the system, it was checked that, by deploying a network of gateways, the LOBIN platform is able to monitor the health status of up to ten patients providing the required QoS (i.e., probability of packet losses lower than 0.2 for each source of traffic). Nevertheless, a more conservative scenario was chosen for the pilot scheme deployed in the Cardiology Unit at La Paz Hospital, where the physiological parameters and location of only five patients were monitored. Deploying a DP network for low-rate applications allows the LOBIN platform to support a much higher number of users.

The autonomy of the developed devices was also checked. WDADs have shown autonomy of 8-9 h, whereas location WTBs are able to work for two days without having to change the batteries.

Finally, the management and control IT application (Server Management and GUIs) was also tested, obtaining positive feedback from the hospital personnel for future applications developments in an operational phase.

\section{ACKNOWLEDGMENT}

The authors would like to thank the rest of the partners involved in the LOBIN project: SIMAVE, NLAZA Soluciones, NUUBO, and GRADIANT, as well as to the Cardiology Unit at La Paz Hospital for the interesting discussions and collaboration during the project.

\section{REFERENCES}

[1] S. J. Palmisano. (2009, Jan. 13). Let's spend on broadband and the power grid. Wall Street J. [Online]. Available: http://online.wsj.com/article/ SB123180687062275609.html

[2] X. F. Teng, Y. T. Zhang, C. C. Y. Poon, and P. Bonato, "Wearable medical systems for p-health," IEEE Rev. Biomed. Eng., vol. 1, pp. 62-74, Dec. $12,2008$.

[3] A. Lymberis and A. Dittmar, "Advanced wearable health systems and applications-Research and development efforts in the European Union," IEEE Eng. Med. Biol. Mag., vol. 26, no. 3, pp. 29-33, May/Jun. 2007. 
[4] U. Anliker, J. A. Ward, P. Lukowicz, G. Tröster, F. Dolveck, M. Baer, F. Keita, E. Schenker, F. Catarsi, L. Coluccini, A. Belardinelli, D. Shklarski, M. Alon, E. Hirt, R. Schmid, and M. Vuskovic, "AMON: A wearable multiparameter medical monitoring and alert system," IEEE Trans. Inf. Technol. Biomed., vol. 8, no. 4, pp. 415-427, Dec. 2004.

[5] C. W. Mundt, K. N. Montgomery, U. E. Udoh, V. N. Barker, G. C. Thonier, A. M. Tellier, R. D. Ricks, R. B. Darling, Y. D. Cagle, N. A. Cabrol, S. J. Ruoss, J. L. Swain, J. W. Hines, and G. T. A. Kovascs, "A multiparameter wearable physiological monitoring system for space and terrestrial applications," IEEE Trans. Inf. Technol. Biomed., vol. 9, no. 3, pp. 382-391, Sep. 2005.

[6] M. D. Rienzo, F. Rizzo, G. Parati, G. Brambilla, M. Ferratini, and P. Castiglioni, "MagIC system: A new textile-based wearable device for biological signal monitoring. Applicability in daily life and clinical settings," in Proc. 27th Ann. Int. Conf. IEEE EMBS, Shanghai, China, Sep. 2005, pp. 7167-7169.

[7] R. Paradiso, G. Loriga, and N. Taccini, "A wearable health care system based on knitted integrated sensors," IEEE Trans. Inf. Technol. Biomed., vol. 9, no. 3, pp. 337-344, Sep. 2005.

[8] V. Shnayder, B. Chen, K. Lorincz, T. R. F. FulfordJones, and M. Welsh, "Sensor networks for medical care," Division Eng. Appl. Sci., Harvard Univ., Cambridge, MA, Tech. Rep. TR-08-05, 2005.

[9] P. S. Pandian, K. Mohanavelu, K. P. Safeer, T. M. Kotresh, D. T. Shakunthala, P. Gopal, and V. C. Padaki, "Smart vest: Wearable multiparameter remote physiological monitoring system," Med. Eng. Phys., vol. 30, no. 4, pp. 466-477, 2007.

[10] Y. D. Lee and W. Y. Chung, "Wireless sensor network based wearable smart shirt for ubiquitous health and activity monitoring," Sens. Actuators B: Chem., vol. 140, pp. 390-395, 2009.

[11] F. Axisa, P. M. Schmitt, G. Delhomme, E. McAdams, and A. Dittmar, "Flexible technologies and smart clothing for citizen medicine, home healthcare, and disease prevention," IEEE Trans. Inf. Technol. Biomed., vol. 9, no. 3, pp. 325-336, Sep. 2005.

[12] M. Pacelli, G. Loriga, N. Taccini, and R. Paradiso, "Sensing fabrics for monitoring physiological and biomechanical variables: E-textile solutions," presented at the 3rd IEEE-EMBS, Boston, MA, 2006.

[13] Textronics, Inc., Official Site. (2010). [Online]. Available: http://www. textronicsinc.com/

[14] V. Zeimpekis, G. M. Giaglis, and G. Lekakos, "A taxonomy of indoor and outdoor positioning techniques for mobile location services," $A C M$ SIGecom Exch., vol. 3, no. 4, pp. 19-27, 2003.

[15] F. Seco, A. R. Jiménez, C. Prieto, J. Roa, and K. Koutsou, "A survey of mathematical methods for indoor localization," in Proc. IEEE Int. Symp. Intell. Signal Process., WISP 2009, pp. 9-14.

[16] A. Anwar, G. Ioannis, and F. N. Pavlidou, "Evaluation of indoor location based on combination of AGPS/HSGPS," in Proc. 3rd Int. Symp. Wireles Pervasive Comput. (ISWPC 2008), pp. 383-387.

[17] S. H. Fang and T. N. Lin, "Accurate WLAN indoor localization based on RSS fluctuations modelling," in Proc. IEEE Int. Symp. Intell. Signal Process., WISP 2009, pp. 27-30.

[18] A. Madhavapeddy and A. Tse, "A study of bluetooth propagation using accurate indoor location mapping," in Proc. Int. Conf. Ubiquitous Comput. (Ubicomp 2005), pp. 105-122.

[19] S. Aparicio, J. Pérez, P. Tarrío, A. M. Bernardos, and J. R. Casar, "An indoor location method based on a fusion map using bluetooth and WLAN technologies," in Proc. Int. Symp. Distrib. Comput. Artif. Intell. 2008 , vol. 50, pp. 702-710, Jan. 2008.

[20] S. P. Subramanian, J. Sommer, F. Zeh, S. Schmitt, and W. Rosenstiel, "PBIL_PDR for scalable bluetooth indoor localization," in Proc. 3rd Int Conf. Exhibition Next Gen. Mobile Appl., Serv. Technol. (NGMAST 2009), pp. $170-175$

[21] F. Guo, C. Zhang, M. Wang, and X. Xu, "Research of indoor location method based on RFID technology," presented at the 11th Joint Conf. Inf. Sci., Shenzhen, China, 2008.

[22] R. Tesoriero, J. Gallud, M. Lozano, and V. Penichet, "Using active and passive RFID technology to support indoor location-aware systems," IEEE Trans. Consum. Electron., vol. 54, no. 2, pp. 578-583, May 2008.

[23] Y. Chu and A Ganz, "A UWB-based 3D location system for indoor environments," presented at the UWBNETS Workshop IEEE BROADNETS Conf., Boston, MA, 2005.

[24] J. Blumenthal, R. Grossman, F. Golatowski, and D. Timmerman, "Weighted centroid localization in Zigbee-based sensor networks," presented at the WISP, 2007. DOI: 10.1109/WISP.2007.4447528.

[25] Wireless Medium Access Control and Physical Layer Specifications for Low-Rate Wireless Personal Area Networks, IEEE Standard 802.15.4, 2003.
[26] Wireless Medium Access Control and Physical Layer Specifications for Low-Rate Wireless Personal Area Networks, IEEE Standard 802.15.4, 2006.

[27] Wireless Medium Access Control and Physical Layer Specifications for Low-Rate Wireless Personal Area Networks, IEEE Standard 802.15.4a, 2007.

[28] P. Baronti, P. Pillai, V. W. C. Chook, S. Chessa, A. Gotta, and Y. F. Hu, "Wireless sensor networks: A survey on the state of the art and the 802.15.4 and Zigbee standards," Comput. Commun., vol. 30, no. 7, pp. 1655-1695, 2007.

[29] K. Akkaya and M. Younis, "A survey on routing protocols for wireless sensor networks," Ad Hoc Netw., vol. 3, no. 3, pp. 325-349, 2005.

[30] J. Sun, Z. Wang, H. Wang, and X. Zhang, "Research on routing protocols based on zigbee network," in Proc. 3rd Int. Conf. Int. Inf. Hiding Multimedia Signal Process. (IIH-MSP 2007), vol. 1, pp. 639-642.

[31] X. Xu, D. Yuan, and J. Wan, "An enhanced routing protocol for zigbee/IEEE 802.15.4 wireless networks," in Proc. 2nd Int. Conf. Future Gen. Commun. Netw. (FGCN 2008), vol. 1, pp. 294-298.

[32] D. Johnson, Y. Hu, and D. Maltz, "The dynamic source routing protocol (DSR) for mobile ad hoc networks for IPv4," RFC 4728 (Experimental), Feb. 2007.

[33] A. Sikora and V. Groza, "Coexistence of IEEE 802.15.4 with other systems in the 2.4 GHz ISM-band," in Proc. IEEE IMTC, May 2005, pp. 17861791.

[34] LOBIN project website. (2008, Jul.). [Online]. Available: http://www. asi-web.net/simave/lobin/

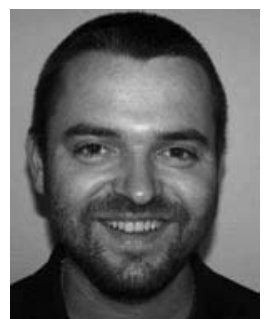

Gregorio López received the Telecommunication Engineering degree in 2008 from Carlos III University of Madrid (UC3M), Madrid, Spain, where he is currently working toward the Ph.D. degree in telematic engineering.

He has been with the UC3M Network Technology Group led by Dr. J. I. Moreno since 2006. During his last semester of 2008, he enjoyed an Erasmus Placement in Deutsche Telekom Laboratories, Berlin, Germany. Some recent research projects in which he has participated are ENERSIP, LOBIN, IRIDIUM, TRANSDATA, Daidalos, and LoRIS. His current research interests include wireless sensor networks (WSNs) and their applications to pervasive healthcare and smart grid and mobility, QoS, and security in next generation networks (NGNs).

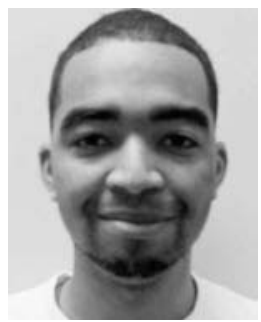

Víctor Custodio received the Computer Engineering degree in 2008 from Carlos III University of Madrid (UC3M), Madrid, Spain.

During his last semester, he combined studying with working in the e-business sector. Since 2008, he has been involved in R\&D projects at UC3M. Some recent Spanish and international research projects in which he has participated are DOMOCELL, LOBIN, IRIDIUM, and TRANSDATA.

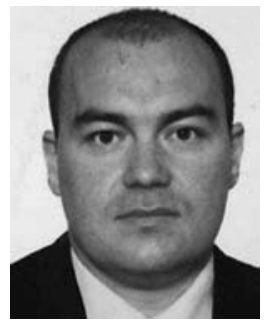

José Ignacio Moreno (M'00) received the Telecommunication Engineering degree and the Ph.D. degree from the Technical University of Madrid (UPM), Madrid, Spain, in 1991 and 1996, respectively.

Since 1997, he has been an Assistant Professor at Carlos III University of Madrid (UC3M), Madrid. He has been involved in several international research projects related to protocol design, protocol engineering, network management, advanced networks, and wireless systems. Some recent research projects in which he has participated are ENERSIP, LOBIN, IRIDIUM, Emagerit, Casertel-NGN, Daidalos, and Moby Dick. He has authored or coauthored more than 80 papers in the field of advanced communications in technical books, journals, and conferences. 